\title{
Penehyclidine mitigates postoperative nausea and vomiting and intraoperative oculocardiac reflex in patients undergoing strabismus surgery: a prospective, randomized, double-blind comparison
}

\author{
Jiacheng Sun ${ }^{\dagger}$, Xiaofei $\mathrm{CaO}^{\dagger}$, Ting Lu, Nan Li, Xinxu Min and Zhengnian Ding ${ }^{*}$ (D)
}

\begin{abstract}
Background: Postoperative nausea and vomiting (PONV) is one of the most frequent complications following strabismus surgery. Penehyclidine, an anticholinergic agent, is widely used as premedication. This study investigated the effect of preoperative penehyclidine on PONV in patients undergoing strabismus surgery.

Methods: In this prospective, randomized, double-blind study, patients scheduled for strabismus surgery under general anesthesia were randomly assigned to either penehyclidine $(n=114)$ or normal saline $(n=104)$ group. Penehyclidine was administrated immediately after anesthesia induction, and normal saline was substituted as control. PONV was investigated from 0 to $48 \mathrm{~h}$ after surgery. Intraoperative oculocardiac reflex (OCR) was also recorded.

Results: Compared with normal saline, penehyclidine significantly reduced PONV incidence (30.7\% vs. 54.8\%, $P<0.01)$ and mitigated PONV severity as indicated by severity scoring $(P<0.01)$. Compared with normal saline, penehyclidine also significantly reduced OCR incidence $(57.9 \%$ vs. $77.9 \%, P<0.01)$ and mitigated OCR severity, as indicated by the requirement for atropine rescue $(77.3 \%$ vs. $90.1 \%, P<0.05)$ and the maximum decrease of heart rate during OCR $(23.1 \pm 9.4 \mathrm{bpm}$ vs. $27.3 \pm 12.4 \mathrm{bpm}, P<0.05)$. The recovery course did not differ between groups. Conclusions: Penehyclidine administrated after anesthesia induction significantly reduced the incidence of PONV and alleviated intraoperative OCR in patients undergoing strabismus surgery.
\end{abstract}

Trial registration: ClinicalTrials.gov (NCT04054479). Retrospectively registered August 13, 2019.

Keywords: Postoperative nausea and vomiting (PONV), Oculocardiac reflex (OCR), Penehyclidine, Strabismus surgery, Anesthesia

\footnotetext{
* Correspondence: zhengnianding@njmu.edu.cn

† Jiacheng Sun and Xiaofei Cao contributed equally to this work. Department of Anesthesiology and Perioperative Medicine, First Affiliated Hospital with Nanjing Medical University, Nanjing 210029, China
}

(c) The Author(s). 2021 Open Access This article is licensed under a Creative Commons Attribution 4.0 International License, which permits use, sharing, adaptation, distribution and reproduction in any medium or format, as long as you give appropriate credit to the original author(s) and the source, provide a link to the Creative Commons licence, and indicate if changes were made. The images or other third party material in this article are included in the article's Creative Commons licence, unless indicated otherwise in a credit line to the material. If material is not included in the article's Creative Commons licence and your intended use is not permitted by statutory regulation or exceeds the permitted use, you will need to obtain permission directly from the copyright holder. To view a copy of this licence, visit http://creativecommons.org/licenses/by/4.0/. The Creative Commons Public Domain Dedication waiver (http://creativecommons.org/publicdomain/zero/1.0/) applies to the data made available in this article, unless otherwise stated in a credit line to the data. 


\section{Background}

Strabismus surgery is a common ophthalmic surgical procedure, especially in pediatric patients. Postoperative nausea and vomiting (PONV) and intraoperative oculocardiac reflex (OCR) are the most frequent complications in patients undergoing strabismus surgery [1, 2]. The incidence of PONV has been shown between 38 and $68.2 \%$ in pediatrics and $45.2 \%$ in adults following strabismus surgery [3-6]. Besides unpleasant experience and delayed discharge, PONV can lead to postoperative complications including fluid and electrolyte imbalances, suture tension, esophageal tear, increased intracranial pressure and pulmonary aspiration [1, 7]. OCR, also known as the Aschner reflex, is defined as a decrease in the heart rate (HR) by greater than $20 \%$ following eyeball pressure or traction of extraocular muscles [8]. The incidence of OCR during strabismus surgery ranges from 14 to $90 \%$ in previous reports [9]. The reflex commonly results in bradycardia and associates with reduced arterial pressure, arrhythmia, asystole, and even cardiac arrest [10]. Both OCR and PONV are the main concerns in patients undergoing strabismus surgery [11].

Penehyclidine is an anticholinergic agent with an elimination half-life over $10 \mathrm{~h}$. As a selective blocker of type 1 and type 3 muscarinic acetylcholine receptor, penehyclidine is widely used as premedication to reduce salivary secretion $[12,13]$. Type 3 and type 5 muscarinic acetylcholine receptors have been reported being associated with the development of motion sickness, a risk factor for PONV [14]. Type 1 muscarinic acetylcholine receptor presents at a high level in vestibular system, and anticholinergics block cholinergic transmission from the vestibular nuclei to advanced central nervous system as well as from the medullary reticular formation to the vomiting center [15]. Considering that the muscarinic acetylcholine receptors are involved in the development of PONV through multiple mechanisms, it is possible that penehyclidine may play a role in preventing PONV in patients undergoing strabismus surgery.

This prospective, randomized, double-blind study was designed to identify whether penehyclidine reduces the incidence of nausea and vomiting in patients after strabismus surgery.

\section{Methods}

This study was prospectively approved by the Human Research Ethics Committee of the First Affiliated Hospital with Nanjing Medical University (\#2019-SR238) and a written informed consent was obtained from patient or legal guardian. During the period of 5 months from July to November 2019, a total of 228 consecutive patients aged $3 \sim 65$ years, scheduled for strabismus surgery under general anesthesia with American Society of Anesthesiologists physical status I and II were enrolled into the present investigation. All methods were performed in accordance with the relevant guidelines and regulations. This manuscript adheres to the applicable CONSORT 2010 guidelines. The patients with obvious vital organ diseases, motion sickness, previous PONV history, smoking, medication with steroids or proton pump inhibitors, or the patients who did not cooperate with intravenous cannulation were excluded. All cases were prospectively and randomly divided into penehyclidine group and normal saline (NS) group. The primary outcome of our study was the effect of penehyclidine on the incidence of nausea and vomiting during the first 48 $\mathrm{h}$ after surgery. The secondary outcome was the possible effect of penehyclidine on the occurrence of intraoperative OCR.

\section{Study protocol \\ Anesthesia induction}

The patients were fasted for over $6 \sim 8 \mathrm{~h}$ as a routine, and monitored with ECG, $\mathrm{SpO}_{2}$ and non-invasive blood pressure as admitted. After venous access established, lactated Ringer's solution was infused at a rate of $10 \sim 15 \mathrm{ml} \cdot \mathrm{kg}^{-1}$. $\mathrm{hr}^{-1}$ throughout anesthesia. Patients were induced with propofol $1.5 \sim 2.5 \mathrm{mg} \cdot \mathrm{kg}^{-1}$ and fentanyl $5.0 \mu \mathrm{g} \cdot \mathrm{kg}^{-1}$. Cisatracurium $0.15 \mathrm{mg} \cdot \mathrm{kg}^{-1}$ was used to facilitate tracheal intubation. No midazolam or inhalational anesthetic agents were used. Lungs were ventilated with a tidal volume of 7 $\sim 10 \mathrm{ml} \cdot \mathrm{kg}^{-1}$ and a frequency of $10 \sim 22$ times per minute to maintain end-tidal $\mathrm{CO}_{2}$ at the level of $35 \sim 40 \mathrm{mmHg}$. The fraction of inspired oxygen was maintained at $60 \%$.

\section{Anesthesia maintenance}

The anesthesia was maintained with infusion of propofol at a rate of $60 \sim 200 \mu \mathrm{g} \cdot \mathrm{kg}^{-1} \cdot \mathrm{min}^{-1}$ and remifentanil at a rate of $0.1 \sim 0.15 \mu \mathrm{g} \cdot \mathrm{kg}^{-1} \cdot \mathrm{min}^{-1}$. No muscle relaxant was further added.

\section{Anesthesia recovery}

Propofol and remifentanil infusion were discontinued as surgery completed. All the patients were spontaneously recovered without the use of neostigmine or atropine to reverse residual muscle relaxant. During the recovery period, the patient who complained of severe pain was treated with parecoxib at a dose of $1.0 \mathrm{mg} \cdot \mathrm{kg}^{-1}$ with an upper limit to $40 \mathrm{mg}$.

\section{Penehyclidine treatment}

Simple randomization was achieved by lottery method and the patient was randomly allocated into penehyclidine or NS group with equal chance. Intravenous penehyclidine at the dose of $10 \mu \mathrm{g} \cdot \mathrm{kg}^{-1}$ with an upper limit to $0.5 \mathrm{mg}$ was administrated immediately after anesthesia induction, and an equal volume of NS served as control. The lots were prepared by a resident, and penehyclidine 
or NS was given by an attending doctor according to the randomization. Patients were unaware of the treatment groups.

\section{Postoperative nausea and vomiting (PONV)}

Nausea and vomiting were investigated after tracheal extubation by a resident who was blinded to the treatment. The occurrence of PONV was recorded by direct interview with patient or legal guardian in hospital and by telephone interview after discharge. The severity of PONV was scored using a numeric rank scoring system according to previous method [16]. The scoring system was composed of four levels: $0=$ no nausea or vomiting; $1=$ nausea but no vomiting; $2=$ vomiting once or twice; $3=$ vomiting on more than two occasions. The patients with severe PONV were treated with granisetron at a dose of $50 \mu \mathrm{g} \cdot \mathrm{kg}^{-1}$ with an upper limit to $3.0 \mathrm{mg}$.

The PONV incidences in different periods, calculated as the percentage of patients who experienced PONV to total number during a specific period, were also analyzed. Some patients may be involved in multiple calculations if they experienced PONV in more than one period.

\section{Oculocardiac reflex (OCR)}

OCR was defined as a decrease in the heart rate by greater than $20 \%$ following eyeball pressure or traction of extraocular muscles [8]. Once OCR was observed, the operation was paused to relieve OCR, and restarted when HR returned to the baseline value. If HR did not recover in $30 \mathrm{~s}$ or severe bradycardia $(\mathrm{HR}<60 \mathrm{bpm}$ for aged $3 \sim 7$ years, and $<50 \mathrm{bpm}$ for more than 7 years old) sustained for over $10 \mathrm{~s}$, intravenous atropine $10 \mu \mathrm{g} \cdot \mathrm{kg}^{-1}$ with an upper limit to $0.5 \mathrm{mg}$ was administrated.

\section{Sample size estimation and statistical analysis}

Sample size evaluation was performed using PASS software, version11 (NCSS, LLC, Kaysville, UT, USA). On the assumption that the use of penehyclidine will result in a decrease in PONV incidence from 50\% in NS group to $30 \%$, 94 cases were needed for each group $(\alpha=0.05$ and $1-\beta=0.8)$. With an anticipation of $10 \% \sim 15 \%$ dropout, at least 105 cases were required for each group.

Data analysis was performed using IBM SPSS software, version 24.0 (IBM Corp., Armonk, NY, USA). Continuous variables conforming to normal distribution were expressed as mean \pm standard deviation and Student's two-tailed unpaired $t$-test was used for comparison between two groups. Non-normal continuous variables were expressed as median (interquartile range, IQR) and Mann-Whitney U test was used for comparison between two groups. Categorical variables were expressed as number and percentage, and analyzed by Fisher's exact test. For ranked data, Mann-Whitney U test was used for comparison. Univariable logistic regression was used to identify the potential risk factors that might affect PONV incidence, and the factors with $P<0.10$ were included in the multivariable logistic regression. A $P$ value $<0.05$ was considered to be significant.

\section{Results}

\section{Patients in the investigation}

During the study period, 228 patients were enrolled into the investigation. Among them, 10 patients dropped out due to lost contact or due to using sevoflurane induction in pediatrics who did not cooperate with intravenous cannulation. Finally, 218 patients were analyzed. The consort flow diagram was shown in Fig. 1.

Between penehyclidine and normal saline groups, patients showed comparable general characteristics, including age, gender, body weight, body height, duration of surgery, duration of anesthesia, unilateral or bilateral operation of eye, and number of operated muscles (Table 1).

\section{Penehyclidine reduced the incidence and mitigated the severity of PONV in patients undergoing strabismus surgery}

As shown in Fig. 2a, the overall incidence of PONV was $30.7 \%$ (35/114) in penehyclidine group and 54.8\% (57/ $104)$ in NS group $(P<0.01)$. Penehyclidine reduced PONV incidence by $44.0 \%$. Penehyclidine significantly mitigated the severity of PONV, as indicated by the numeric rank scoring system, compared with NS $(P<0.01)$ (Fig. 2b).

\section{The anti-PONV effect of penehyclidine over time Nausea and vomiting showed high incidence within $6 \mathrm{~h}$ after tracheal extubation}

To observe the dynamic change of PONV, the PONV incidence during $0 \sim 2,2 \sim 6,6 \sim 24$ and $24 \sim 48 \mathrm{~h}$ after tracheal extubation were analyzed. In NS group, the PONV incidence was $40.4 \%$ (42/104) during the first $2 \mathrm{~h}$ and remained as high as $35.6 \%(37 / 104)$ during the next period from 2 to $6 \mathrm{~h}$ after tracheal extubation. The PONV incidence robustly and significantly decreased during the periods from 6 to $24 \mathrm{~h}(8.7 \%, 9 / 104)$ and 24 to $48 \mathrm{~h}(1.0 \%, 1 / 104)$ thereafter, when compared with the incidence during 2 to $6 \mathrm{~h}$ after tracheal extubation $(P<0.01)$ (Fig. 3).

\section{Penehyclidine significantly reduced the incidence of nausea and vomiting within $6 \mathrm{~h}$ after tracheal extubation}

In penehyclidine group, the incidence of nausea and vomiting were $25.4,18.4,5.3$ and $0 \%$ in the periods of the first $2 \mathrm{~h}, 2 \sim 6,6 \sim 24$ and $24 \sim 48 \mathrm{~h}$ after tracheal extubation, respectively. PONV incidence in the periods of the first $2 \mathrm{~h}$ and $2 \sim 6 \mathrm{~h}$ were significantly reduced by 


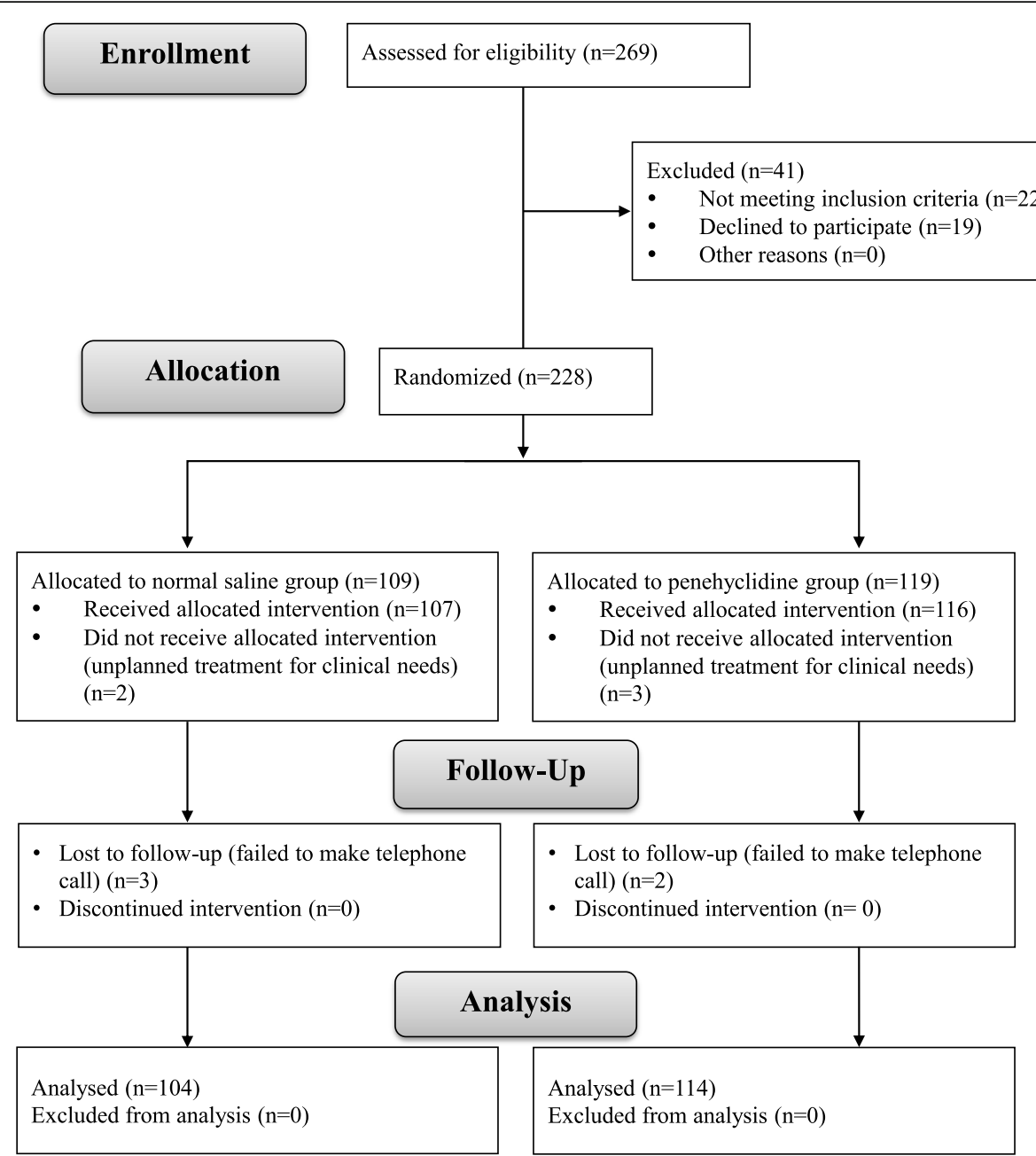

Fig. 1 Consort flow diagram. Total 228 patients were randomly allocated to penehyclidine or normal saline group. Among them, 10 patients dropped out due to lost contact or using inhalation anesthesia induction in pediatric patients who did not cooperate with intravenous induction. Finally, 218 patients were analyzed ( $n=104$ in normal saline group and $n=114$ in penehyclidine group)

Table 1 General characteristics of patients

\begin{tabular}{llll}
\hline Parameters & Normal saline $(\boldsymbol{n}=\mathbf{1 0 4})$ & Penehyclidine $(\boldsymbol{n}=\mathbf{1 1 4})$ & $\boldsymbol{P}$ value \\
\hline Age (year) & $10(11)$ & $11(14)$ & 0.398 \\
Gender (Male/Female) & $61 / 43$ & $57 / 57$ & 0.222 \\
Body weight (kg) & $42.5(33.4)$ & $47.5(33.3)$ & 0.412 \\
Body height (cm) & $150(43)$ & $155(35)$ & 0.656 \\
Duration of surgery (min) & $28(16)$ & $30(16)$ & 0.668 \\
Duration of anesthesia (min) & $64(17.3)$ & $63.5(20)$ & 0.631 \\
Unilateral/bilateraloperation & $18 / 86$ & $19 / 95$ & 1.000 \\
Number of operated muscles & $3(2)$ & $3(2)$ & 0.753 \\
\hline
\end{tabular}

Age, body weight, body height, duration of surgery, duration of anesthesia and number of operated muscles are expressed as medium (IQR), and compared by Mann-Whitney $\mathrm{U}$ test between normal saline group and penehyclidine group. Gender is expressed as numbers of male/female patients and unilateral/bilateral operation is expressed as numbers of the respective patients. Fisher's exact test was used for comparison between normal saline group and penehyclidine group 

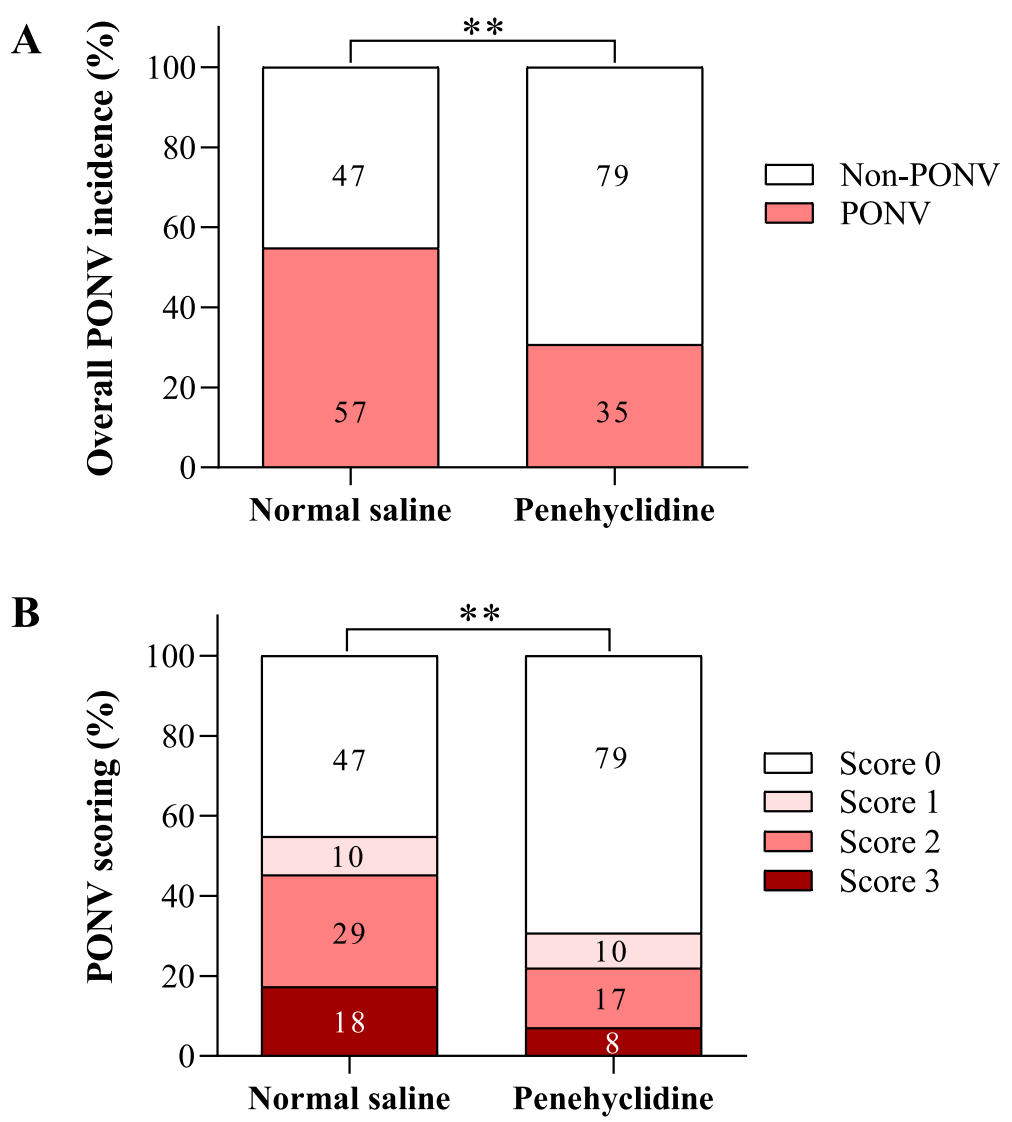

Fig. 2 Penehyclidine reduced the incidence and mitigated the severity of PONV in patients undergoing strabismus surgery. a The overall incidence of PONV were recorded within $48 \mathrm{~h}$ after tracheal extubation. $\mathbf{b}$ The severity of PONV was scored using a numeric rank scoring system ranging from 0 to 3, wherein 0 represented no nausea and no vomiting and 3 represented vomiting on more than two occasions. ${ }^{* *} P<0.01$ analyzed by Fisher's exact test (a) or by Mann-Whitney $U$ test $(\mathbf{b})$. The numbers of patients are shown in the figures. PONV, postoperative nausea and vomiting

37.0 and $48.2 \%$ when compared with the time-matched PONV incidence in NS group $(P<0.05$ or 0.01$)$ (Fig. 3). No significant difference in PONV incidence was observed between penehyclidine group and NS group in the periods of $6 \sim 24$ and $24 \sim 48 \mathrm{~h}$.

\section{Penehyclidine independently reduced PONV risk}

To exclude the confounding factors which potentially interfere the anti-PONV effect of penehyclidine, logistic regression analysis was conducted. Multivariable logistic regression showed that penehyclidine was an independent protective factor (Odds Ratio: 0.330, 95\% confidence interval: $0.178 \sim 0.609, P<0.01)$. Operation with $4 \sim 6$ muscles independently increased PONV risk (Odds Ratio: 3.553, 95\% confidence interval: $1.909 \sim 6.615, P<0.01)$. Gender, age, occurrence of OCR, duration of surgery, and duration of anesthesia were not associated with PONV risk in this study (Table 2).
Penehyclidine reduced the incidence and severity of oculocardiac reflex during strabismus surgery

The incidence of OCR was $77.9 \%$ (81/104) in NS group and $57.9 \%(66 / 114)$ in penehyclidine group during strabismus surgery $(P<0.01)$, which was reduced by $25.7 \%$ (Fig. 4a). In patients with OCR, atropine rescue was used in $90.1 \%(73 / 81)$ of patients in NS group and $77.3 \%$ (51/ $66)$ in penehyclidine group $(P<0.05)$, which was reduced by $14.2 \%$ (Fig. 4 b). Penehyclidine significantly reduced the maximum decrease of heart rate during OCR by $15.4 \%(23.1 \pm 9.4 \mathrm{bpm} \quad$ vs. $27.3 \pm 12.4 \mathrm{bpm}$, $P<0.05)$ (Fig. 4c).

\section{Penehyclidine did not affect the recovery course after anesthesia}

The time from the end of surgery to tracheal extubation, length of stay in the post-anesthesia care unit, use of antiemetics and analgesics, occurrence of severe dry mouth, facial flush, and drowsiness are shown in Table 3. No significant difference was detected between groups. 


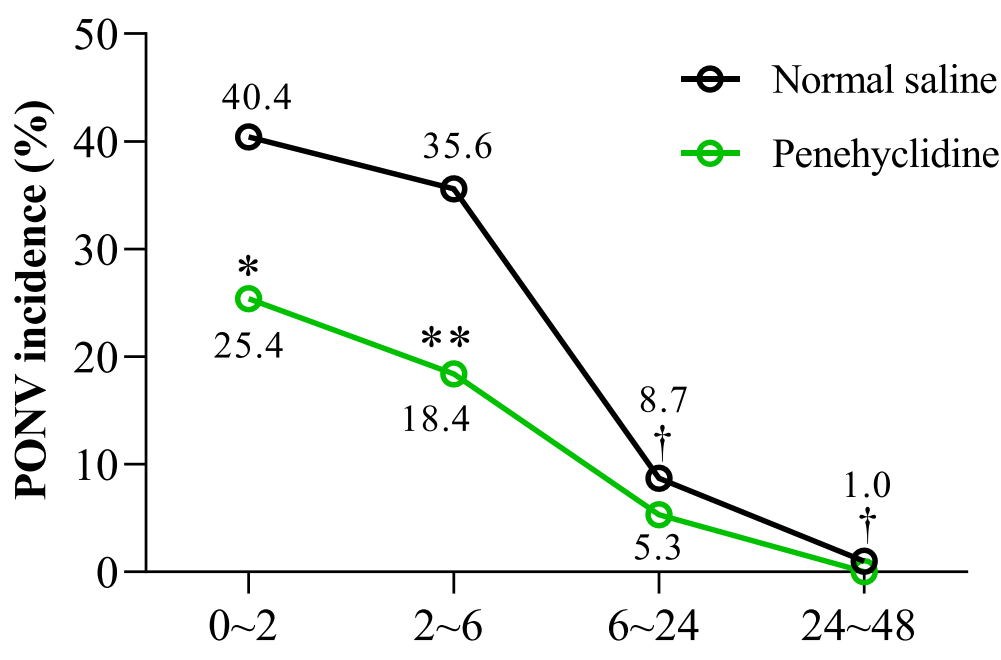

Hours postoperation

Fig. 3 The anti-PONV effect of penehyclidine over time. The PONV incidences in different postoperative periods are shown in the figure. ${ }^{\dagger} P<0.01$ vs. the incidence during $2 \sim 6 \mathrm{~h}$ in normal saline group; ${ }^{* *} P<0.01$ and ${ }^{*} P<0.05$ vs. the time-matched normal saline group, analyzed by Fisher's exact test. PONV, postoperative nausea and vomiting

\section{Discussion}

The main finding in this study is that penehyclidine administration after anesthesia induction significantly mitigated the incidence and severity of both postoperative nausea and vomiting and intraoperative oculocardiac reflex in patients undergoing strabismus surgery.

PONV is a common complication after general anesthesia and strabismus surgery. The risk factors for the development of PONV relate to patients, anesthetic techniques, and type of surgery $[17,18]$. Female, non-smokers, history of PONV or motion sickness, and use of opioids are the most common risk factors [18]. The inhalational anesthetics, ketamine, and etomidate increase the incidence of PONV while the use of propofol, midazolam and free fluid infusion technique are believed to reduce its incidence $[1,19]$. For underage patients, duration of surgery over $30 \mathrm{~min}$, age over 3 years and receiving strabismus surgery are independent risk factors for PONV [20, 21]. Many drugs have been used for the management of PONV and the most widely used are 5-hydroxytryptamine (5- $\left.\mathrm{HT}_{3}\right)$ receptor antagonists. The Neurokinin-1 receptor antagonists, corticosteroids, butyrophenone and antihistamines are also recommended. However, antiemetic agents raise different concerns just like the risk of QT prolongation in $5-\mathrm{HT}_{3}$ receptor antagonists and the effect on postoperative infection as well as blood glucose levels in

Table 2 Univariable and multivariable logistic regression analysis

\begin{tabular}{|c|c|c|c|c|c|}
\hline \multirow[t]{2}{*}{ Variables } & & \multicolumn{2}{|l|}{ Univariable Analysis } & \multicolumn{2}{|l|}{ Multivariable Analysis } \\
\hline & & Odds Ratio (95\% Cl) & $P$ value & Odds Ratio $(95 \% \mathrm{Cl})$ & $P$ value \\
\hline \multirow[t]{2}{*}{ Gender } & Male & - & - & & \\
\hline & Female & $1.146(0.668 \sim 1.965)$ & 0.621 & & \\
\hline \multirow[t]{2}{*}{ Age (years) } & $3 \sim 17$ & - & - & & \\
\hline & $18 \sim 65$ & $0.540(0.288 \sim 1.011)$ & 0.054 & $0.750(0.377 \sim 1.489)$ & 0.410 \\
\hline \multirow[t]{2}{*}{ EOM operated (n) } & $1 \sim 3$ & - & - & & \\
\hline & $4 \sim 6$ & $3.344(1.906 \sim 5.868)$ & $<0.01$ & $3.553(1.909 \sim 6.615)$ & $<0.01$ \\
\hline Oculocardiac reflex & & $2.224(1.216 \sim 4.068)$ & $<0.01$ & $1.558(0.807 \sim 3.006)$ & 0.186 \\
\hline Penehyclidine & & $0.365(0.210 \sim 0.636)$ & $<0.01$ & $0.330(0.178 \sim 0.609)$ & $<0.01$ \\
\hline Duration of surgery (min) & & $1.012(0.991 \sim 1.035)$ & 0.263 & & \\
\hline Duration of anesthesia (min) & & $1.007(0.991 \sim 1.022)$ & 0.394 & & \\
\hline
\end{tabular}

Univariable logistic regression was performed first and the factors with $P<0.10$ were included in the multivariable logistic regression. As a result, age, extraocular muscles operated, occurrence of oculocardiac reflex and use of penehyclidine were selected for multivariable logistic regression. $\mathrm{Cl}$ Confidence interval, EOM Extraocular muscles 
A

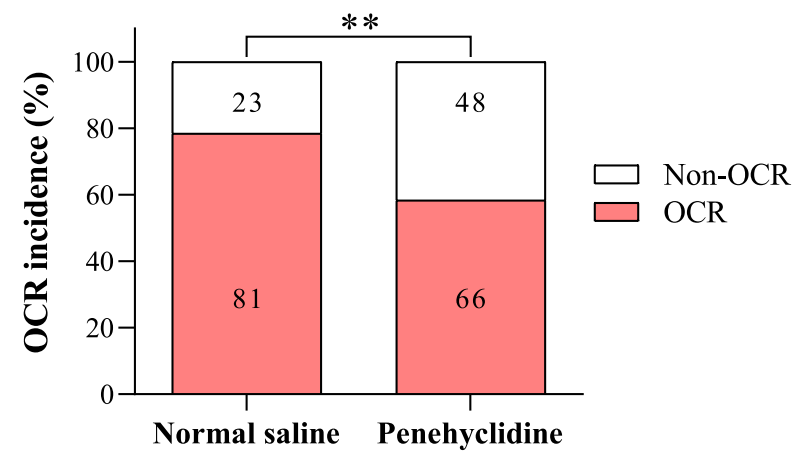

B

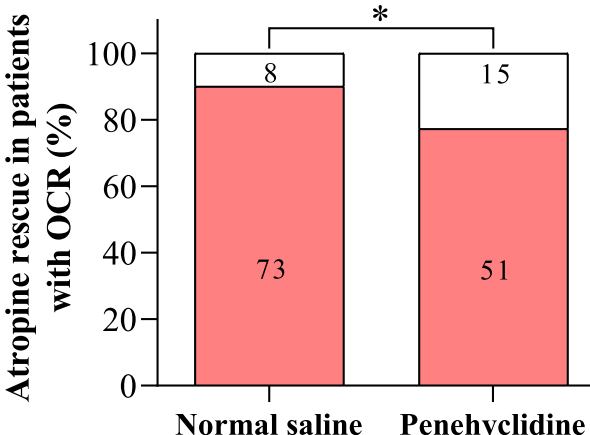

Atropine wasn't

needed for rescure

Atropine was

needed for rescure

C

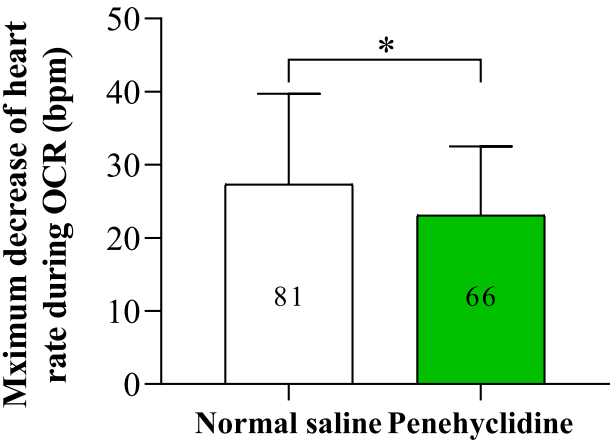

Fig. 4 Penehyclidine reduced the incidence and severity of OCR during strabismus surgery. a The incidence of OCR in each group. $\mathbf{b}$ The requirement for atropine rescue in patients with OCR. $\mathbf{c}$ The maximum decrease of heart rate during $O C R .{ }^{* *} P<0.01$ and ${ }^{*} P<0.05$ analyzed by Fisher's exact test $(\mathbf{a}, \mathbf{b})$ or by unpaired student $t$-test $(\mathbf{c})$. The numbers of patients are shown in the figures. The maximum decreases of heart rate are shown as means \pm standard deviation. OCR, oculocardiac reflex; bpm, beats per minute

Table 3 Postoperative recovery

\begin{tabular}{llll}
\hline & Normal saline $(\boldsymbol{n}=\mathbf{1 0 4})$ & Penehyclidine $(\boldsymbol{n}=\mathbf{1 1 4})$ & $\boldsymbol{P}$ value \\
\hline Antiemetics used & $4(3.9 \%)$ & $1(0.9 \%)$ & 0.195 \\
Analgesics used & $1(1 \%)$ & $3(2.7 \%)$ & 0.623 \\
Time to extubation $(\mathbf{m i n})$ & $25(16)$ & $25(13)$ & 0.922 \\
Length of stay in PACU $(\mathbf{m i n})$ & $59(30)$ & $60(30)$ & 0.732 \\
Severe dry mouth & 0 & 0 & - \\
Facial flushing & $1(1 \%)$ & $1(0.9 \%)$ & 1.000 \\
Drowsiness & 0 & $1(0.9 \%)$ & 1.000 \\
\hline
\end{tabular}

Requirement for antiemetics and analgesics, severe dry mouth, facial flushing and drowsiness are expressed as number (\%), and compared by Fisher's exact test between normal saline group and penehyclidine group. Time to extubation and length of stay in PACU are expressed as medium (IQR) and compared by MannWhitney $U$ test between groups. PACU, post-anesthesia care unit 
corticosteroids [21, 22]. Here in this study, penehyclidine, an anticholinergic agent, significantly reduced the incidence of PONV in patients undergoing strabismus surgery.

The incidence of PONV following strabismus surgery was found to be $54.8 \%$ in normal saline group in our result, which is consistent with previous reports. It was also found that PONV mainly occurred in the early recovery period. Penehyclidine administration after anesthesia induction significantly reduced PONV incidence and mitigated its severity.

Unexpectedly, the use of penehyclidine alleviated oculocardiac reflex during strabismus surgery, while penehyclidine was considered having no obvious effect on heart rate [12]. Once stimulated by manipulation, the ophthalmic branch of the trigeminal nerve transports the sensory message to central nervous system, causing impulses exiting the brainstem and transmit to the sinoatrial node. This activates the vagal motor response and ultimately leading to sinus bradycardia, atrioventricular block, ventricular ectopy, ventricular fibrillation or even asystole [10]. Several approaches can be applied to relieve or prevent OCR. Pausing the surgery immediately upon OCR eliminates the pressure on the eyeball or the traction of extraocular muscles, which can alleviate the reflex [10]. Repeated pauses may disturb the process of surgery. Preoperative atropine or glycopyrrolate can attenuate the negative effect of vagus nerve on heart rate during OCR through blocking peripheral type 2 muscarinic receptors in the heart $[10,23]$. The main side effect of both agents is undesirable dysrhythmia such as sinus tachycardia. In this study, penehyclidine reduced the incidence of OCR by $25.7 \%$, the requirement for atropine rescue by $14.2 \%$, and the maximum decrease of heart rate by $15.4 \%$. It is unclear whether the effect of penehyclidine on OCR is caused by its intrinsic type 2 muscarinic receptor block effect or its central effects.

The main side effects of penehyclidine include dry mouth and central anticholinergic syndrome, similar to other anticholinergics [15]. Its central sedative effect may delay anesthesia recovery. In our investigation, a dose of $10 \mu \mathrm{g} \cdot \mathrm{kg}^{-1}$ penehyclidine with an upper limit to $0.5 \mathrm{mg}$ was used. The time to tracheal extubation and the length of stay in the post-anesthesia care unit were found comparable between penehyclidine and NS group. No patient complained of severe dry mouth and no patient developed central anticholinergic syndrome postoperatively. These may be attributed to the limited maximal dose, and that the patients could drink freely after minor surgery [24].

The agents including midazolam, etomidate, neostigmine or inhalational anesthetics were not used in this study in order to avoid potential effects on PONV. The randomization and double-blind technique were strictly followed during the investigation.

\section{Conclusions}

This study identified penehyclidine, a widely used preoperative anticholinergic agent, as an effective protector against postoperative nausea and vomiting and intraoperative oculocardiac reflex in patients undergoing strabismus surgery.

\section{Abbreviations}

PONV: Postoperative nausea and vomiting; OCR: Oculocardiac reflex; ECG: Electrocardiograph; $\mathrm{SpO}_{2}$ : Saturation of pulse oxygen; bpm: Beats per minute; IQR: Interquartile range; 5- $\mathrm{HT}_{3}$ : 5-hydroxytryptamine; NS: Normal saline; EOM: Extraocular muscles

\section{Acknowledgements}

Not applicable.

\section{Authors' contributions}

ZD contributed to study conception and design, interpretation of data, and drafted the article. JS, XC, TL and NL contributed to clinical investigation and data collection. JS and XM contributed to statistical analysis. All authors have read and approved the manuscript.

\section{Funding}

National Natural Science Foundation of China (81870234) and Jiangsu Province's Outstanding Medical Academic Leader program (15) provided funding for this work. The funders had no role in study design, data collection, analysis and interpretation, and in writing the manuscript.

\section{Availability of data and materials}

The data sets generated and analyzed during the current study are not publicly available due to the stipulations of ethics committee to protect individual privacy of patients but are available from the corresponding author on reasonable request.

\section{Ethics approval and consent to participate}

This study was prospectively approved by the Human Research Ethics Committee of the First Affiliated Hospital with Nanjing Medical University (\#2019-SR-238) and a written informed consent was obtained from patient or legal guardian.

\section{Consent for publication}

Not applicable.

\section{Competing interests}

The authors declared that they have no competing interests.

Received: 28 August 2020 Accepted: 4 February 2021

Published online: 13 February 2021

\section{References}

1. Sayed JA, MA FR. MO MA. Comparison of dexamethasone or intravenous fluids or combination of both on postoperative nausea, vomiting and pain in pediatric strabismus surgery. J Clin Anesth. 2016;34:136-42.

2. Kim SH, Shin HJ. Effects of an infratrochlear nerve block on reducing the oculocardiac reflex during strabismus surgery: a randomized controlled trial. Graefes Arch Clin Exp Ophthalmol. 2018;256(9):1777-82.

3. Chen JY, Jia JE, Liu TJ, et al. Comparison of the effects of dexmedetomidine, ketamine, and placebo on emergence agitation after strabismus surgery in children. Can J Anaesth. 2013;60(4):385-92.

4. Wolf R, Morinello E, Kestler G, et al. PONV after strabismus surgery: risk adapted prophylaxis? Anaesthesist. 2016;65(7):507-13.

5. Joo J, Park S, Park HJ, et al. Ramosetron versus ondansetron for postoperative nausea and vomiting in strabismus surgery patients. BMC Anesthesiol. 2016;16(1):41. 
6. Shen Y, Chen C, Wu C, et al. Dexamethasone, ondansetron, and their combination and postoperative nausea and vomiting in children undergoing strabismus surgery: a meta-analysis of randomized controlled. trials. 2014;24(5):490-8.

7. Son J, Yoon H. Factors affecting postoperative nausea and vomiting in surgical patients. J Perianesth Nurs. 2018;33(4):461-70.

8. Lai Y, Hsu H, Wang H, et al. The oculocardiac reflex during strabismus surgery: its relationship to preoperative clinical eye findings and subsequent postoperative emesis. Journal of AAPOS : the official publication of the American Association for Pediatric Ophthalmology. Strabismus. 2014;18(2): 151-5.

9. Ha SG, Huh J, Lee BR, et al. Surgical factors affecting oculocardiac reflex during strabismus surgery. BMC Ophthalmol. 2018;18(1):103.

10. Dunville LM, Sood G, Kramer J. Oculocardiac Reflex. Treasure Island: StatPearls; 2020

11. Rodgers A, Cox R. Anesthetic management for pediatric strabismus surgery: continuing professional development. Can J Anaesth. 2010;57(6):602-17.

12. Wang Y, Gao Y, Ma J, et al. Pleiotropic effects and pharmacological properties of penehyclidine hydrochloride. Drug Design Dev. 2018;12:3289-99.

13. Xiao $\mathrm{H}$, Liao Z, Tong $\mathrm{R}$, et al. Penehyclidine hydrochloride: a potential drug for treating COPD by attenuating toll-like receptors. Drug Design Dev. 2012; 6:317-22.

14. Horn CC, Wallisch WJ, Homanics GE, et al. Pathophysiological and neurochemical mechanisms of postoperative nausea and vomiting. Eur J Pharmacol. 2014;722:55-66.

15. Pergolizzi J, Philip B, Leslie J, et al. Perspectives on transdermal scopolamine for the treatment of postoperative nausea and vomiting. J Clin Anesth. 2012;24(4):334-45.

16. Oh AY, Kim JH, Hwang JW, et al. Incidence of postoperative nausea and vomiting after paediatric strabismus surgery with sevoflurane or remifentanil-sevoflurane. Br J Anaesth. 2010;104(6):756-60.

17. Apfel C, Kranke P, Eberhart L. Comparison of surgical site and patient's history with a simplified risk score for the prediction of postoperative nausea and vomiting. Anaesthesia. 2004;59(11):1078-82.

18. Pierre S, Benais H, Pouymayou J. Apfel's simplified score may favourably predict the risk of postoperative nausea and vomiting. Can J Anaesth. 2002; 49(3):237-42.

19. Scheiermann P, Herzog F, Siebenhofer A, et al. Intravenous versus inhalational anesthesia for pediatric inpatient surgery - a systematic review and meta-analysis. J Clin Anesth. 2018;49:19-25.

20. Eberhart $\mathrm{LH}$, Geldner $\mathrm{G}$, Kranke P, et al. The development and validation of a risk score to predict the probability of postoperative vomiting in pediatric patients. Anesth Analg. 2004;99(6):1630-7 table of contents.

21. Gan T, Belani K, Bergese $\mathrm{S}$, et al. Fourth consensus guidelines for the Management of Postoperative Nausea and Vomiting. Anesth Analg. 2020; 131(2):411-48

22. Yokoi A, Mihara T, Ka K, et al. Comparative efficacy of ramosetron and ondansetron in preventing postoperative nausea and vomiting: an updated systematic review and meta-analysis with trial sequential analysis. PLoS One. 2017;12(10):e0186006.

23. Chisakuta AM, Mirakhur RK. Anticholinergic prophylaxis does not prevent emesis following strabismus surgery in children. Paediatr Anaesth. 1995;5(2): 97-100.

24. Zhang Z, Zhuang $Y$, Ouyang F, et al. Penehyclidine enhances the efficacy of tropisetron in prevention of PONV following gynecological laparoscopic surgery. J Anesth. 2012;26(6):864-9.

\section{Publisher's Note}

Springer Nature remains neutral with regard to jurisdictional claims in published maps and institutional affiliations.

Ready to submit your research? Choose BMC and benefit from:

- fast, convenient online submission

- thorough peer review by experienced researchers in your field

- rapid publication on acceptance

- support for research data, including large and complex data types

- gold Open Access which fosters wider collaboration and increased citations

- maximum visibility for your research: over $100 \mathrm{M}$ website views per year

At $\mathrm{BMC}$, research is always in progress.

Learn more biomedcentral.com/submissions 